\title{
Effect of different surgical procedures on the accuracy of prediction of the plasma concentration of fentanyl: comparison between mastectomy and laparoscopic prostatectomy
}

Yoshihito Fujita ${ }^{1,2^{*}}$ (D), Saya Yoshizawa ${ }^{2}$, Maiko Hoshika² ${ }^{2}$ Koichi Inoue ${ }^{3}$, Shoko Matsushita ${ }^{3}$, Hisao Oka ${ }^{3}$ and Kazuya Sobue ${ }^{2}$

\begin{abstract}
Background: The accuracy of simulation-predicted fentanyl concentration in different types of surgical procedure is not fully understood. We wished to estimate the effect of different types of surgical procedure on the accuracy of such simulations.

Findings: Fifty patients who had undergone elective mastectomy or laparoscopic prostatectomy (American Society of Anesthesiologists physical status = I-II) were enrolled. Anesthesia was maintained throughout surgery with sevoflurane and a bolus infusion of fentanyl. A maintenance infusion was administered with $8 \mathrm{~mL} / \mathrm{kg} / \mathrm{h}$ Ringer's acetate solution from the start of anesthesia to completion of blood sampling. An infusion to compensate for blood loss was administered (one to two volumes of hydroxyethyl starch). A blood sample was drawn every 30 min during anesthesia.

We measured the plasma concentration of fentanyl in 358 samples from 50 patients. The plasma concentration of fentanyl was correlated significantly with the simulated predicted fentanyl concentration $(r=0.734, P<0.01)$ but $36.0 \%$ of all samples had a difference greater than $\pm 0.5 \mathrm{ng} / \mathrm{mL}$. Approximately $0.3 \mathrm{ng} / \mathrm{mL}$ of a fixed bias was shown throughout mastectomy. During laparoscopic prostatectomy, the fixed bias gradually became negative from $\approx 0.3$ to $-0.3 \mathrm{ng} / \mathrm{mL}$ as the sampling stage proceeded.

Conclusions: The predicted concentration of fentanyl was significantly correlated with the plasma concentration of fentanyl $(r=0.734)$. However, there were different patterns of a fixed bias between mastectomy and laparoscopic prostatectomy groups. We should pay attention to this tendency among different surgical procedures.

Trial registration: UMIN000005110

Keywords: Fentanyl, Concentration, Simulation, Prediction
\end{abstract}

\footnotetext{
* Correspondence: fujita.yoshihito.823@mail.aichi-med-u.ac.jp

${ }^{1}$ Department of Anesthesiology, Aichi Medical University School of Medicine,

1-1 Karimata Yazako, Nagakute, Aichi 480-1195, Japan

${ }^{2}$ Department of Anesthesiology and Intensive Care Medicine, Nagoya City

University Graduate School of Medical Sciences, 1 Kawasumi, Mizuho-cho,

Mizuho-ku, Nagoya, Japan

Full list of author information is available at the end of the article
} 


\section{Findings}

Introduction

Fentanyl is a synthetic opioid used widely to supplement general anesthesia. Fentanyl is administered in general anesthesia upon prediction of its concentration with a simulator. The Shafer model is used worldwide [1]. This predictive model is applied for various types of surgical procedure. Recently, interest has focused on which other factors may be responsible for alterations in pharmacokinetics with the objective of reducing variability and increasing accuracy. Kazama and colleagues, using a pseudo-steady-state model of propofol, found that compensated hemorrhage increased the propofol concentration by 20\%; however, if uncompensated shock was induced, the propofol concentration increased by $375 \%$ [2]. Using a non-steady-state model, Egan and colleagues found that hemorrhagic shock reduced central clearance as well as the central volumes of fentanyl [3] and remifentanil, resulting in a higher fentanyl concentration in animals suffering from shock. Other factors, such as age $[4,5]$, sex $[6,7]$, cardiac output $[8,9]$, and obesity [10], were also investigated.

However, the accuracy of the simulator in different surgical procedures is not fully understood. In the present study, we chose mastectomy and laparoscopic prostatectomy because the former has a short operative time and low blood loss, whereas the latter has a relatively long operative time and several hundred blood loss. We investigated the effect of these different surgical procedures on the accuracy of simulation-predicted fentanyl concentration in plasma.

\section{Materials and methods}

Approval (number 528) of the study protocol by the Research Ethics Board was obtained, as was written informed consent from patients. This study is registered with the University Hospital Medical Information Network Clinical Trials Registry (UMIN-CTR, UMIN000005110).

Patients graded as class I or II according to the classification set by the American Society of Anesthesiologists undergoing mastectomy or laparoscopic prostatectomy were enrolled. Patients with the following conditions were excluded: (i) American Society of Anesthesiologists physical status class III, IV, or V; (ii) age $<19$ or $>71$ years; (iii) body mass index $>30 \mathrm{~kg} / \mathrm{m}^{2}$; (iv) height $>181 \mathrm{~cm}$; and (v) weight $>80 \mathrm{~kg}$.

Anesthesia was induced with propofol $(1.5-2.0 \mathrm{mg} / \mathrm{kg}$ body weight), fentanyl (1-3 $\mu \mathrm{g} / \mathrm{kg})$, and a bolus infusion of rocuronium $(1 \mathrm{mg} / \mathrm{kg})$. Anesthesia throughout the surgical procedure was maintained with sevoflurane and fentanyl after a bolus infusion of propofol and rocuronium. After the initial dose of fentanyl, a bolus infusion of fentanyl $(25,50$, or $100 \mu \mathrm{g} /$ bolus $)$ was adjusted by the participating anesthesiologist to meet the clinical need in each situation. Anesthesia was titrated subsequently to a bispectral index of $\approx 50$. Continuous intravenous infusion of Ringer's acetate solution was started in the operating room. A maintenance infusion was administered with $8 \mathrm{~mL} / \mathrm{kg} / \mathrm{h}$ Ringer's acetate solution from the start of anesthesia until blood samples had been obtained. In addition, infusion for blood loss was administered with one to two volumes of $6 \%$ hydroxyethyl starch (70/0.5). Pharmacokinetic simulations were not used to guide anesthesia: they were carried out after the cases had been completed.

Blood samples from an arterial catheter were taken every $30 \mathrm{~min}$ from the initial administration of fentanyl to $30 \mathrm{~min}$ after the end of the surgical procedure. Samples were placed immediately on ice and centrifuged at $1000 \times g$ and $4^{\circ}$ for $5 \mathrm{~min}$ within $1 \mathrm{~h}$, and the plasma was stored at $-80{ }^{\circ} \mathrm{C}$ until analyses. Samples taken within $10 \mathrm{~min}$ of the bolus infusion of fentanyl were excluded from analyses.

Quantification of fentanyl in plasma was done using online solid-phase extraction liquid chromatography coupled with mass spectrometry (Micromass Quattro Premier; Waters, Milford, MA, USA) in multiplereaction monitoring mode [11]. The monitoring ions were $m / z 337.1 \rightarrow 187.9$ for fentanyl and $m / z 342.1 \rightarrow$ 187.9 for fentanyl- $\mathrm{d}_{5}$ (internal standard), respectively.

Table 1 Characteristics of the patients

\begin{tabular}{|c|c|c|}
\hline & Mastectomy & $\begin{array}{l}\text { Laparoscopic } \\
\text { prostatectomy }\end{array}$ \\
\hline & Number $=30$ & Number $=20$ \\
\hline Age (years) & $52.3 \pm 9.7$ & $66(63,70)[45,70]$ \\
\hline Sex & Female & Male \\
\hline Height (cm) & $156.6 \pm 5.0$ & $168.3 \pm 6.5$ \\
\hline Weight (kg) & $52.9 \pm 7.7$ & $64.1 \pm 9.3$ \\
\hline AHA physical status I, II & 22,8 & 8,12 \\
\hline Operation time (min) & $115.1 \pm 35.9$ & $\begin{array}{l}179.5(163,300) \\
{[139,344]}\end{array}$ \\
\hline Anesthesia time (min) & $160.8 \pm 39.5$ & $\begin{array}{l}251.5(219,364.5) \\
{[195,407]}\end{array}$ \\
\hline Fentanyl ( $\mu \mathrm{g})$ & $566.0 \pm 169.0$ & $\begin{array}{l}900(812.5,900) \\
{[500,1700]}\end{array}$ \\
\hline Number of samples & 176 & 182 \\
\hline \multicolumn{3}{|l|}{ Infusion } \\
\hline$A R$ & $941.3 \pm 381.1$ & $\begin{array}{l}1750(1525,2465) \\
{[1050,3200]}\end{array}$ \\
\hline HES & $0(0,0)[0,1000]$ & $\begin{array}{l}600(425,1650) \\
{[200,2700]}\end{array}$ \\
\hline Blood loss & $24(5,44)[5,679]$ & $\begin{array}{l}563.5(400,739) \\
{[52,2550]^{\mathrm{a}}}\end{array}$ \\
\hline
\end{tabular}

${ }^{\mathrm{a} B l o o d}$ loss in laparoscopic prostatectomy includes urine from the surgical field $A R$ acetate Ringer's solution, HES hydroxyethyl starch 
The lower limit of quantitation of fentanyl in human plasma was $0.05 \mathrm{ng} / \mathrm{mL}$. Recovery values ranged from 99 to $111 \%$ for inter-day (relative standard deviation: $1.7-6.9 \%)$ and intra-day assays (1.0-10.1\%). This method was applied for measurement of the fentanyl level in plasma from patients.

The pharmacokinetic model described by Shafer was used to predict the plasma concentration of fentanyl. In conjunction with the administration schedule for each patient, the Tivatrainer program (www.eurosiva.eu/tivatrainer/TTweb/ TTinfo.html) was used to generate the predicted fentanyl concentration in plasma for the Shafer model [1].

The present study was designed to detect a correlation between the predicted and measured concentrations of fentanyl in plasma. The effect size was a correlation coefficient $(r)>0.5$. Power analyses indicated that a minimum of 38 samples would be needed to detect this correlation with a power of $90 \%$ and an $\alpha$ of 0.05 . We estimated that, to analyze three to four subgroups, we would need $\approx 160$ samples (38 multiplied by 4) for the mastectomy and laparoscopic prostatectomy groups. Continuous variables are presented as the mean $\pm \mathrm{SD}$ or median (interquartile range) depending on the normality of the distribution as confirmed by the Shapiro-Wilk test.

\section{Results}

Fifty patients were recruited between February and November 2011. Thirty patients undergoing mastectomy and 20 patients undergoing laparoscopic prostatectomy completed the study with 358 usable data. The cardiac, renal, and liver functions of all patients were normal. The fentanyl concentration in the plasma of 410 samples was measured. However, 48 samples of control data from before fentanyl addition and four samples taken within $10 \mathrm{~min}$ of the bolus infusion of fentanyl were excluded because of inappropriate timing. Baseline characteristics are shown in Table 1.

The correlation between the plasma concentration of fentanyl and predicted plasma concentration of fentanyl is shown in Fig. 1. The plasma concentration of fentanyl was correlated significantly with the predicted concentration $(r=0.734, P<0.01, y=0.94 \mathrm{x}+0.29)$. The difference in the fentanyl concentration between plasma and the simulation for the 358 samples is shown in Fig. 2 . The difference between the plasma concentration of fentanyl and simulation-predicted fentanyl concentration was $0.19 \pm 0.54$ [range, -1.52 to 1.67 ]. In addition, $36.0 \%$ of all samples had a difference of more than $\pm 0.5 \mathrm{ng} / \mathrm{mL}$. The difference between the plasma concentration of

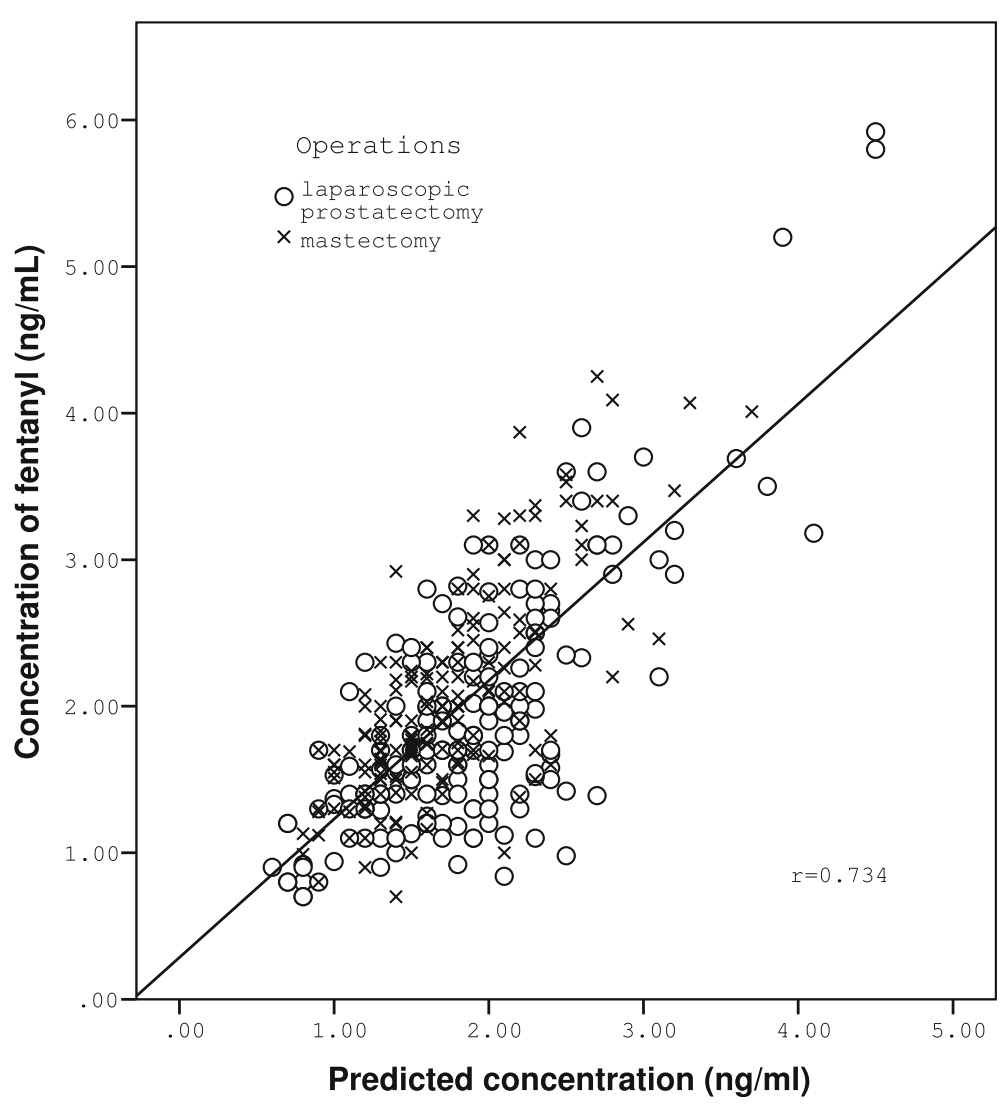

Fig. 1 Correlation between the plasma concentration of fentanyl and simulation-predicted concentration of fentanyl. The plasma concentration of fentanyl was correlated significantly with the simulation-predicted concentration of fentanyl $(r=0.734, P<0.01, y=0.94 x+0.29)$ 


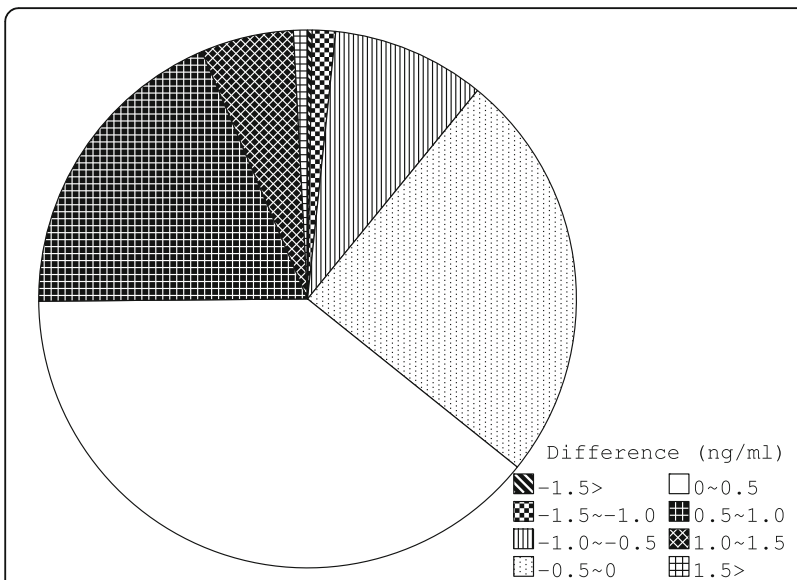

Fig. 2 Difference in the fentanyl concentration between plasma and simulation for 358 samples. Among these samples, 36.0\% had a difference of greater than $\pm 0.5 \mathrm{ng} / \mathrm{mL}$

fentanyl and simulation-predicted fentanyl concentration plotted against time from the first administration of fentanyl is shown in Fig. 3. There was an effect of time from the first administration of fentanyl on the difference between the plasma concentration of fentanyl and simulation-predicted fentanyl concentration $(r=-0.293$, $P<0.01, y=-0.02 x+0.429)$.

We wished to examine the relationship between the different surgical procedures and the influence of different sampling times. Hence, sampling data were divided into four groups at 90-min intervals for each type of surgical procedure. Samples of arterial blood were obtained within $1.5 \mathrm{~h}$ from the initial administration of fentanyl (stage 1), during 1.5 to $3 \mathrm{~h}$ (stage 2), during 3 to $4.5 \mathrm{~h}$ (stage 3), and after $4.5 \mathrm{~h}$ (stage 4). Interestingly, our data showed that the tendency of a fixed bias differed between mastectomy and laparoscopic prostatectomy. In mastectomy, the difference between the measured fentanyl concentration and simulation-predicted fentanyl concentration had no correlation with time from the first administration of fentanyl $(r=0.157, \quad P<0.01, \quad y=0.01 x+$ 0.203 ). Approximately $0.3 \mathrm{ng} / \mathrm{mL}$ of a fixed bias was shown in all sampling stages (Fig. 4a). However, in laparoscopic prostatectomy, a fixed bias was influenced by the sampling stage (Fig. 4b) and gradually became negative as the sampling stage increased. More details are shown in Additional file 1.

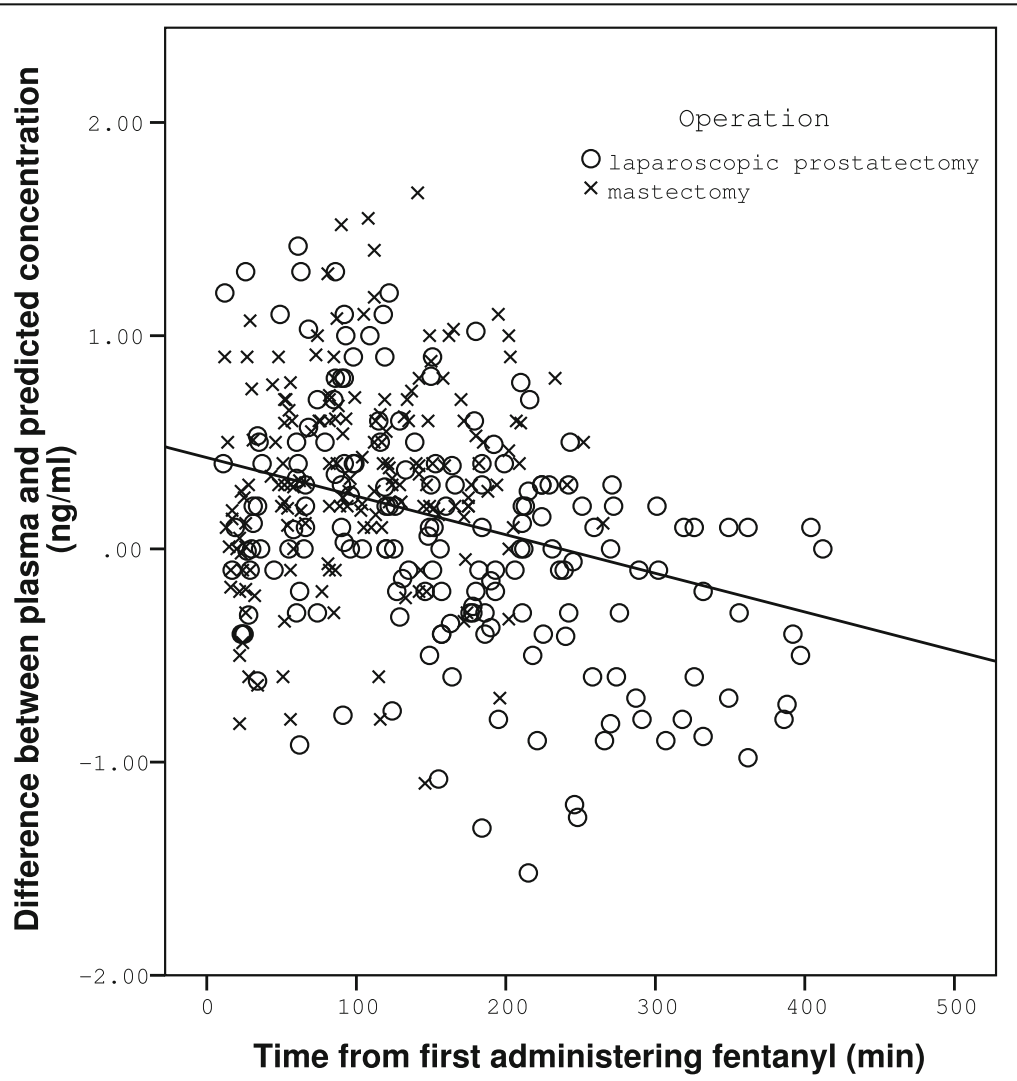

Fig. 3 Difference between the plasma concentration of fentanyl and simulation-predicted concentration of fentanyl plotted against time from the first administration of fentanyl. There was an effect of time from the first administration of fentanyl on the difference between the plasma concentration of fentanyl and simulation-predicted fentanyl concentration $(r=-0.293, P<0.01, y=-0.02 x+0.429)$ 

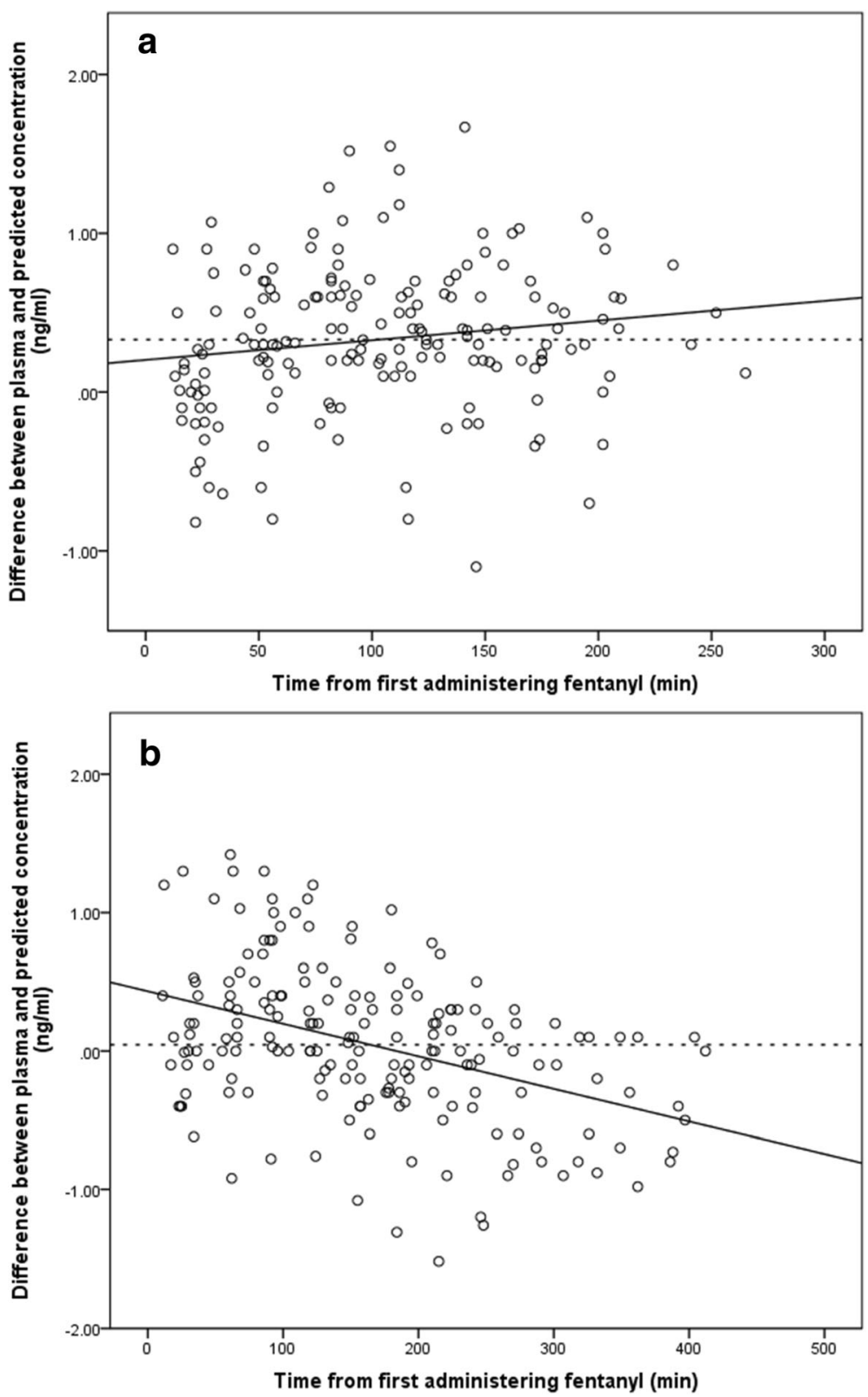

Fig. 4 Difference in the predictive accuracy between mastectomy and laparoscopic prostatectomy. In mastectomy, the difference between the measured concentration of fentanyl and simulation-predicted concentration of fentanyl had no correlation with time from the first administration of fentanyl $(r=0.157, P<0.01, y=0.01 x+0.203)$. Approximately $0.3 \mathrm{ng} / \mathrm{mL}$ of a fixed bias was shown in all sampling stages (a). However, in the laparoscopic prostatectomy group, a fixed bias was influenced by sampling stage $(\mathbf{b})$, and gradually became negative as the sampling stage increased. This finding implies that $\approx 0.3 \mathrm{ng} / \mathrm{mL}$ of a fixed bias in stage 1 decreased to $-0.3 \mathrm{ng} / \mathrm{mL}$ in stage 4 systematically $(r=-0.411, P<0.01, y=-0.02 x+0.432)$

\section{Discussion}

In the present study, we demonstrated that the predicted data were significantly correlated with the plasma concentration of fentanyl $(r=0.734)$. However, a different pattern of a fixed bias appeared between mastectomy and laparoscopic prostatectomy. In mastectomy, $\approx 0.3 \mathrm{ng} / \mathrm{mL}$ of a fixed bias was shown in all sampling stages. However, in laparoscopic prostatectomy, a fixed bias became increasingly negative from $\approx 0.3$ to $-0.3 \mathrm{ng} / \mathrm{mL}$ as the sampling stage proceeded. Hence, anesthesiologists should pay attention to this difference among different surgical procedures.

In a clinical setting, the primary concern has been how far the measured concentration is from the predicted concentration [1]. The accuracy of simulation has been described most frequently in terms of the "performance error." That is, the difference between the measured concentration and target concentration as a 
percentage of the desired target (i.e., [measured - target]/target $\times 100 \%$ ) [12]. However, in the present study, the difference in the fixed bias due to the type of surgical procedure revealed that the median absolute performance error did not show this tendency (Table 2). In a clinical setting, the accuracy of the performance error might not be sufficient to detect a difference between the predicted fentanyl concentration and measured fentanyl concentration.

There were differences between mastectomy and laparoscopic prostatectomy in terms of patient age, sex, operative time, blood loss, volume of infusion, and other factors (Table 1). One of the reasons for the difference in the volume of infusion might have been dilution of the administered fluid compensating for blood loss. Blood loss in laparoscopic prostatectomy was greater than that in mastectomy (Table 1). In our protocol, the maintenance infusion remained constant, and infusion for blood loss was administered with one to two volumes of hydroxylethyl starch according to the clinical strategy. We considered that increasing the infusion for blood loss might have caused a reduction in the plasma concentration of fentanyl. Several authors have concluded that hemorrhage increases the drug concentration $[2,3]$. Those results are not in accordance with the results in the present study. In our study, patients were administered a sufficient volume to avoid hemorrhagic shock. In addition, we assumed that, in those studies, hemorrhagic shock itself induced a reduction in fentanyl clearance, for example, by reducing central clearance [3]. Without shock status, the fentanyl concentration might be influenced directly by dilution of the infusion.

Several studies have investigated the effect of age on pharmacokinetic parameters $[4,5]$. Pharmacokinetic parameters change in the elderly, but whether these changes are sufficiently different to affect the accuracy of measurement of these parameters has not been established. In our study, the patients in the laparoscopic prostatectomy group were older than those in the mastectomy group. In general, elderly patients may be considered to have a lower clearance of fentanyl. In addition, this scenario might cause a higher concentration of fentanyl during laparoscopic prostatectomy. Nevertheless, lower concentrations compared with simulation were shown during laparoscopic prostatectomy. This factor might not have influenced the results of this study. We considered that the age difference might not have affected the outcomes of our study markedly.

Cardiac output has been shown to affect the early pharmacokinetics of fentanyl distribution [8, 9]. Higher cardiac output has been shown to reduce the arterial concentration of fentanyl compared with that in a model

Table 2 Analyses of each sampling stage

\begin{tabular}{|c|c|c|c|c|}
\hline Sampling stage & Stage 1 & Stage 2 & Stage 3 & Stage 4 \\
\hline Sampling time & $<1.5 \mathrm{~h}$ & $1.5-3 \mathrm{~h}$ & $3-4.5 \mathrm{~h}$ & $>4.5 \mathrm{~h}$ \\
\hline \multicolumn{5}{|l|}{ Mastectomy } \\
\hline Number of samples & 83 & 74 & 19 & 0 \\
\hline Plasma fentanyl concentration (ng/mL) & $2.08 \pm 0.74$ & $2.07 \pm 0.70$ & $1.88 \pm 0.54$ & \\
\hline Simulated fentanyl concentration ( $\mathrm{ng} / \mathrm{mL}$ ) & $1.81 \pm 0.54$ & $1.68 \pm 0.52$ & $1.50 \pm 0.31$ & \\
\hline The difference between plasma and predicted fentanyl & $0.27 \pm 0.48$ & $0.38 \pm 0.47$ & $0.34 \pm 0.44$ & \\
\hline Range of difference & {$[-0.82,1.52]$} & {$[-1.1,1.68]$} & {$[-0.7,1.1]$} & \\
\hline Number of a difference more than $+0.5 \mathrm{ng} / \mathrm{mL}$ & 28/83 (38\%) & $27 / 74(36 \%)$ & $6 / 19(32 \%)$ & \\
\hline Number of a difference less than $-0.5 \mathrm{ng} / \mathrm{mL}$ & $6 / 83(7 \%)$ & $3 / 74(4 \%)$ & $1 / 19(5 \%)$ & \\
\hline Median absolute performance error (\%) & 21 & 25 & 35 & \\
\hline Fixed bias [95\% confidence interval] (ng/mL) & $0.27[0.170 .37]$ & $0.38\left[\begin{array}{lll}0.27 & 0.49\end{array}\right]$ & $0.38\left[\begin{array}{lll}0.17 & 0.59\end{array}\right]$ & \\
\hline \multicolumn{5}{|l|}{ Laparoscopic prostatectomy } \\
\hline Number of samples & 48 & 59 & 48 & 27 \\
\hline Plasma fentanyl concentration (ng/mL) & $2.27 \pm 1.26$ & $2.08 \pm 0.63$ & $1.68 \pm 0.56$ & $1.43 \pm 0.43$ \\
\hline Simulated fentanyl concentration $(\mathrm{ng} / \mathrm{mL})$ & $1.98 \pm 1.04$ & $1.90 \pm 0.47$ & $1.83 \pm 0.55$ & $1.76 \pm 0.48$ \\
\hline The difference between plasma and predicted fentanyl & $0.29 \pm 0.54$ & $0.19 \pm 0.52$ & $-0.15 \pm 0.53$ & $-0.34 \pm 0.41$ \\
\hline Range of difference & {$[-0.92,1.42]$} & {$[-1.08,1.20]$} & {$[-1.52,0.78]$} & {$[-0.98,0.30]$} \\
\hline Number of a difference more than $+0.5 \mathrm{ng} / \mathrm{mL}$ & $13 / 48(27 \%)$ & $14 / 59(26 \%)$ & $2 / 48(4 \%)$ & $0 / 27(0 \%)$ \\
\hline Number of a difference less than $-0.5 \mathrm{ng} / \mathrm{mL}$ & $2 / 48(4 \%)$ & $5 / 59(8 \%)$ & $10 / 48(21 \%)$ & $12 / 48(25 \%)$ \\
\hline Median absolute performance error (\%) & 18 & 18 & 20 & 21 \\
\hline Fixed bias [95\% confidence interval] (ng/mL) & $0.29[0.140 .44]$ & $0.18\left[\begin{array}{ll}0.03 & 0.33\end{array}\right]$ & $-0.15[0-0.30]$ & $-0.34[-0.17-0.51]$ \\
\hline
\end{tabular}


of normal cardiac output [9]. Especially in the laparoscopic prostatectomy group, the administration of hydroxyl ethyl starch for the compensation of blood loss might have increased cardiac output and influenced our results.

The intensity of nociceptive stimulation during a surgical procedure may change the pharmacokinetics of a drug because pain induces vascular contraction via sympathetic nerves. The intensity of nociceptive stimulation might have been different between mastectomy and laparoscopic prostatectomy. Nevertheless, mastectomy and laparoscopic prostatectomy are not strongly invasive types of surgical procedure. Therefore, this factor might not have affected the results of this study markedly.

Our investigation had two main limitations. First, we chose patients who had undergone mastectomy or laparoscopic prostatectomy. The data obtained did not cover simulations of all types of surgical procedure. In addition, using data from different types of surgical procedure besides mastectomy and laparoscopic prostatectomy would have been beneficial. Second, opioid doses are often based on the total body weight of a patient. In the present study, the total body weight of almost all patients was in the normal range (Table 1). Thus, our data cannot be extrapolated to lean or obese patients.

\section{Conclusion}

We demonstrated a variation in the difference between the plasma concentration of fentanyl and predicted concentration of fentanyl in a clinical setting. The predicted concentration of fentanyl was significantly correlated with the plasma concentration of fentanyl, with $r=0.734$. However, there were several patterns of fixed bias between the mastectomy and laparoscopic prostatectomy groups. Our results suggest that the type of surgical procedure should be considered when predicting the concentration of an anesthetic agent.

\section{Additional file}

Additional file 1: Supplementary files. (ZIP $97 \mathrm{~kb}$ )

\section{Acknowledgements}

We thank Drs Megumi Harima, Kazuma Fujikake, Naoko Takeuchi, Min-Hye So, Shinichiro Yoshimura, Yoshiki Sento, Satoshi Aoki, Yukiko Mori, Taiki Kojima, Kentaro Miyake, and Toshihiro Yasui for helping to collect blood samples and acquire data.

\section{Funding}

Support was obtained solely from institutional and departmental sources.

\section{Availability of data and materials}

The datasets supporting the conclusions of this article are available in the UMIN-ICDR repository UMIN000005110 http://www.umin.ac.jp/icdr/index.html.

\section{Authors' contributions}

YF, SY, and MH participated in the design of the study and undertook statistical analyses. YF drafted the manuscript. KI, SM, and $\mathrm{HO}$ carried out the measurements of fentanyl in plasma. YS, SY, and MH collected the blood samples, acquired the patient data, and analyzed the data. $\mathrm{HO}$ and KS conceived the study, participated in its design and coordination, and helped to draft the manuscript. All authors approved the final version of the manuscript.

\section{Competing interests}

The authors declare that they have no competing interests.

\section{Consent for publication}

Written informed consent was obtained from the patients' parents for publication.

\section{Ethics approval and consent to participate}

All procedures undertaken in studies involving human participants were in accordance with the ethical standards of our institutional research committee and the 1964 Helsinki declaration and its later amendments or comparable ethical standards. The Research Ethics Board of Nagoya City University approved the study protocol (number 528). This study is registered with the University Hospital Medical Information Network Clinical Trials Registry (UMIN-CTR, UMIN000005110). Written informed consent was obtained from all individual participants included in the study.

\section{Previous presentation in congress}

2012 Annual Meeting of the European Anesthesiology in Paris, France, 9-12 June 2012

\section{Publisher's Note}

Springer Nature remains neutral with regard to jurisdictional claims in published maps and institutional affiliations.

\section{Author details}

'Department of Anesthesiology, Aichi Medical University School of Medicine, 1-1 Karimata Yazako, Nagakute, Aichi 480-1195, Japan. 'Department of Anesthesiology and Intensive Care Medicine, Nagoya City University Graduate School of Medical Sciences, 1 Kawasumi, Mizuho-cho, Mizuho-ku, Nagoya, Japan. ${ }^{3}$ Department of Physical and Analytical Chemistry, School of Pharmacy, Kinjo Gakuin University, 2-1723 Omori, Moriyama, Nagoya 463-8521, Japan.

Received: 6 February 2017 Accepted: 2 May 2017

Published online: 19 May 2017

\section{References}

1. Shafer SL, Varvel JR, Aziz N, Scott JC. Pharmacokinetics of fentanyl administered by computer-controlled infusion pump. Anesthesiology. 1990;73:1091-102.

2. Kazama T, Kurita T, Morita K, Nakata J, Sato S. Influence of hemorrhage on propofol pseudo-steady state concentration. Anesthesiology. 2002;97:1156-61.

3. Egan TD, Kuramkote S, Gong G, Zhang J, McJames SW, Bailey PL. Fentanyl pharmacokinetics in hemorrhagic shock: a porcine model. Anesthesiology. 1999:91:156-66.

4. Ginsberg B, Howell S, Glass PS, Margolis JO, Ross AK, Dear GL, et al. Pharmacokinetic model-driven infusion of fentanyl in children. Anesthesiology. 1996:85:1268-75.

5. Scott JC, Stanski DR. Decreased fentanyl and alfentanil dose requirements with age. A simultaneous pharmacokinetic and pharmacodynamic evaluation. J Pharmacol Exp Ther. 1987;240:159-66.

6. Vuyk J, Oostwouder CJ, Vletter AA, Burm AG, Bovill JG. Gender differences in the pharmacokinetics of propofol in elderly patients during and after continuous infusion. Br J Anaesth. 2001:86:183-8.

7. Schuttler J, Ihmsen H. Population pharmacokinetics of propofol: a multicenter study. Anesthesiology. 2000;92:727-38.

8. Henthorn TK, Krejcie TC, Avram MJ. The relationship between alfentanil distribution kinetics and cardiac output. Clin Pharmacol Ther. 1992;52:190-6.

9. Bjorkman S, Wada DR, Stanski DR. Application of physiologic models to predict the influence of changes in body composition and blood flows on the pharmacokinetics of fentanyl and alfentanil in patients. Anesthesiology. 1998:88:657-67

10. Shibutani K, Inchiosa Jr MA, Sawada K, Bairamian M. Accuracy of pharmacokinetic models for predicting plasma fentanyl concentrations in lean and obese surgical 
patients: derivation of dosing weight ("pharmacokinetic mass"). Anesthesiology. 2004;101:603-13.

11. Inoue K, Matsushita S, Nomura M, Tatemathu M, Mizuno K, Hino T, et al. Determination of fentanyl drug in cancer patient serum by liquid chromatography with mass spectrometry. Jpn J Pharm Palliat Care Sci. 2011;:1111-6.

12. Varvel JR, Donoho DL, Shafer SL. Measuring the predictive performance of computer-controlled infusion pumps. J Pharmacokinet Biopharm. 1992;20:63-94.

\section{Submit your manuscript to a SpringerOpen ${ }^{\circ}$ journal and benefit from:}

- Convenient online submission

- Rigorous peer review

- Immediate publication on acceptance

- Open access: articles freely available online

- High visibility within the field

- Retaining the copyright to your article

Submit your next manuscript at $\boldsymbol{\sim}$ springeropen.com 